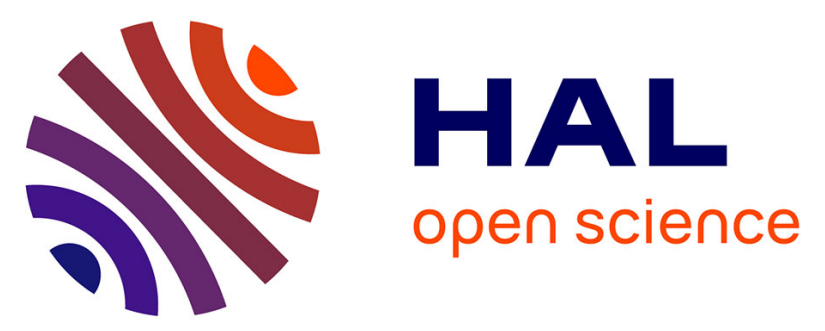

\title{
Multiphase Pipe Flow velocity measurements in Strong Colored Noisy Doppler Ultrasound: Parametric and non parametric approaches
}

Denis Kouamé, Cheick Guetbi, Jean-Pierre Remenieras, Jean-Marc Girault, Abdeldjalil Ouahabi, Frédéric Patat

\section{To cite this version:}

Denis Kouamé, Cheick Guetbi, Jean-Pierre Remenieras, Jean-Marc Girault, Abdeldjalil Ouahabi, et al.. Multiphase Pipe Flow velocity measurements in Strong Colored Noisy Doppler Ultrasound: Parametric and non parametric approaches. IEEE International Ultrasonics Symposium (IUS 1997), Oct 1997, Toronto, Canada. pp.815-818, 10.1109/ULTSYM.1997.663138 . hal-03153375

\section{HAL Id: hal-03153375 https://hal.science/hal-03153375}

Submitted on 2 Mar 2021

HAL is a multi-disciplinary open access archive for the deposit and dissemination of scientific research documents, whether they are published or not. The documents may come from teaching and research institutions in France or abroad, or from public or private research centers.
L'archive ouverte pluridisciplinaire HAL, est destinée au dépôt et à la diffusion de documents scientifiques de niveau recherche, publiés ou non, émanant des établissements d'enseignement et de recherche français ou étrangers, des laboratoires publics ou privés. 


\title{
MULTIPHASE PIPE FLOW VELOCITY MEASUREMENT IN STRONG COLORED NOI- SY DOPPLER ULTRASOUND : PARAMETRIC AND NON PARAMETRIC APPROACHES
}

\author{
D. KOUAMÉ, C. GUETBI, J-P REMENIERAS, J-M GIRAULT, A. OUAHABI, F.PATAT
}

\author{
GIP UItrasons/LUSSI-EIT, 7 Avenue M. Dassault, 37000 Tours, France
}

E-mail : kouame@univ-tours.fr

\begin{abstract}
Real time flow velocity measurement is a problem of considerable practical interest in industrial and biomedical applications. Due to their good frequency resolution, parametric methods such as recursive least squares or their variants are commonly used in such cases. However, some typical problems of these methods, namely correct order selection and fitness of the parametric model, may alter the accuracy of the frequency estimates. This paper provides comparison between specific Instrumental Variable (IV) identification which simultaneously gives model parameters and orders, and different non parametric time-frequency estimators. Results of tests on modeled Doppler signals with known flow velocity profile, are discussed.
\end{abstract}

\section{INTRODUCTION}

Many flow velocity estimation methods for Doppler systems have been widely explored in literature [1-5]. In a real-time two-dimensional (2-D) Doppler system, the velocity estimation is performed with few ( 8 or 16) samples to compare with the image cadence. This has implied the development of fast- but not accurate- algorithms. In the case of precise measurements of flow velocity, it is necessary to find other methods. In practice, the precise determination of the Doppler frequency may be cumbersome because of the various physical parameters which introduce uncertainties in the velocity estimation [1]. In a previous work [8], we show the good performances and the interest of specific factored form of parametric methods for velocimetry.

The purpose of this paper is to provide comparison between these parametric methods and non parametric ultrasound Doppler frequency estimation by use of modeled signal with known physical characteristics. Computer simulations are used in order to perform statistical comparison of the different time varying frequency estimation methods under the same circumstances.

\section{DOPPLER SIGNAL GENERATION AND FREQUENCY MEASUREMENT}

The experimental scheme of Doppler signal measurement which is associated to our simulation is given in Fig. I.



Fig. I Experimental scheme of Doppler signal measurement

Two sets of ultrasound transducers $E$ ( Emission) and R (Reception) are placed across a Plexiglas pipe containing a twophase fluid. The received signal is demodulated and sampled. At each sample time $t_{i}$, the quadrature analytical signal can be written as:

$$
\begin{aligned}
\left.y\left(t_{i}\right)=K\left(t_{i}\right) \exp j\left(\omega_{d}\left(t_{i}\right)+\phi\left(t_{i}\right)\right)\right] \\
\omega_{d}(t)=2 \pi \frac{2 f_{0}}{c} \cos \alpha \int_{0}^{j} v(\tau) d \tau
\end{aligned}
$$

$K\left(t_{i}\right)$ is the random magnitude of the Doppler signal depending on the characteristics of the transducers, $\phi\left(t_{i}\right)$ the random phase depending on the position of the particles of the flowing fluid, and $v(t)$ is the flow velocity to be measured through the Doppler shift pulsation $\omega_{d f}(t), \alpha=\pi / 4$ is the angle between the ultrasound probe axis and the flow direction, $f_{0}=$ $100 \mathrm{kHz}$ is the emission signal frequency, $c$ is the ultrasound celerity. In order to simulate the experimental conditions of the flow velocity measurement, we use the simulation procedure described in [2]. Thus, for a given time velocity profile $v(t)$, we can compute the simulated Doppler signal which is not a simple frequency modulation.

\section{BACKGROUND AND METHODS}

\subsection{Parametric methods : Factored Instrumental Variable (IV)}

This method is applied on a model of the signal rather than the signal itself. In the parametric methods, by using the Doppler signal, the ARX ( or here AR) parameters and the associated power spectral density are first computed, and then Doppler frequency is estimated. In this part, our attention will be focused on the problem of parameters estimation. The following developments are based on the Birman's block data factorization [6], [7] applied to the recursive least squares estimation. Here, 
due to the complex demodulation of the ultrasound signal, the data are complex numbers. Let us first describe the specific complex IV method. Consider the general AR model with complex coefficients:

$$
y(t)=\sum_{i=1}^{n} a_{i} y(t-i)+\xi(t)
$$

where $\xi$ is a complex noise which can be colored and $n$ is assumed to be the maximum possible order of eq. (2). Eq. (2) can be written

$$
\begin{aligned}
& y(t)=h^{T}(t) \theta+\xi(t) \\
& \text { with } h(t)=[-y(t-n), \ldots,-y(t-1)]^{\prime},
\end{aligned}
$$

$\theta=\left\{a_{n,}, a_{1-1}, \ldots, a_{1}\right\}^{\prime}$ and ${ }^{T}$ is the transpose operator.

We recall here the principal steps of the IV algorithm for complex Doppler signal parameter estimation [8].

Let $x(t)$ be the instrument. This instrument accounts for the possible colored noise in the signal.

$x(t)=[-3(t-d-n), \ldots,-y(t-d-1)]^{t} ; d$ is the instrument delay.

and let also $s(t)=[-x(t-t) \ldots . ., x t-2),-x(t-1)]^{7}$

Define $\varphi(t)=\left[h^{\prime}(t),-s(t)\right]^{\prime}, \eta(t)=[\varsigma(t),-x(t)]^{\prime}$ and

$$
C_{n}(1)=\left[\sum_{i}^{\prime} \eta(j) \cdot \varphi^{\prime}(j)\right]_{1 a+\ldots, \ldots+1,}^{\prime}
$$

$C_{n}(t)$ is referred to as covariance matrix. Using the iterative $U D V^{H}$ decomposition, $C(t)$ can be written as follows.

$C_{n}(t)=U_{n}(t) D_{n}(t) V_{n}^{\prime \prime}(t)$ where $U_{n}$ is an upper complex triangular matrix with I on the diagonal, $D_{n}$ a real diagonal matrix and the symbol ${ }^{\mathrm{H}}$ denotes the hermitian transpose. Al the end of the iterations, $U_{n}$ takes the following shape at order $n$ :

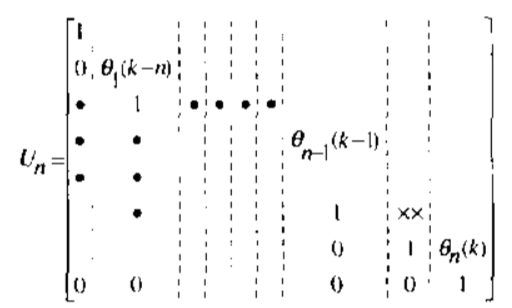

$D_{n}$ is the diagonal matrix written at order $\mathrm{n}$ :

$$
D_{n}=\operatorname{dica}\left[J_{n}^{-1}(k-n) \quad \ldots J_{n-1}^{-1}(k-1) J_{n}^{-1}(k)\right] \text {. (7) }
$$

The $\theta_{i}$ are the estimates of the parameters $\theta$ at the order $i$. Thus we have a recursion based on the order $i=1$ to $n$.

The $J_{i}$ are achieved during the iterative decomposition, and are similar to the Akaike Information Criterion and can be used to select the model order. The recursion on the time $t$ is obtained by writing

$$
C_{n}(t)=U_{n}(t-1)\left[D_{n}(t-1)-\frac{g_{6}, g_{s+1}^{n}}{\beta_{n}(t)}\right] V_{n}^{n}(t-1) .
$$$$
\text { with } \quad f_{\text {un }}=U_{n}{ }^{\prime}(t-1) \varphi(t) \text {, }
$$

$$
g_{*+n}=D_{n}(t-1) \cdot f_{x_{n}}
$$


symbol * denotes the complex conjugation. Doppler frequency is obtained by computing the AR parameters associated to the analytical Doppler signal and then computing the maximum pole frequency of the power spectrum density

$$
P\left(f^{\prime}\right)=\frac{\gamma}{\mid 1+\sum_{i 1}^{n} a_{i} \exp \left(-2 \pi j k f T,\left.\right|^{2}\right.}
$$

where $\gamma$ is a positive constant proportional to the covariance of the innovation of the AR model, $T$, is the sampling period. and $f$ the reduced variable frequency:

$$
-0.5<f<0.5
$$

\subsection{Nonparametric methods : Time-frequency distributions}

In many velocimetry applications, the ultrasonic signal is a broadband pulse modulated at the center frequency of the transducer. Therefore the signal extent is usually finite in both time and frequency domains. Time-frequency distributions are then a useful tool for such analysis. These distributions (which belong to the Cohen class) can be formulated with a unified approach as



where $Q(u, \tau)$ is a function which defines the distribution and its properties, $t$ and $f$ respectively denote the time and frequency components associated to the signal $y$. Further details on such distributions can be found in [10], [11]. Note that the classical Fourier power spectrum density is a special case of these distributions. Due to the well-known limits of the intuitive Fourier analysis, more convenient time-frequency estimators have received considerable amount of attention in frequency estimation.

\section{The Wigner-Ville Distributions(WVD)}

The WVD is another special case of these distributions.

We consider here one variant of these distributions (since the other variants do not give significantly different results) in the case of Doppler signal frequency estimation.

For an analytical signal y the continuous WVD is given by:

$$
W(t, f)=\int x(t+\tau / 2) y(t-\tau / 2) \exp (-j 2 \pi \tau) d \tau
$$
signal.

$W y(t, f)$ gives for each time, the frequency components of the

\subsection{The Wavelet Transforms(WT)}

An extension of the previous case is obtained by defining the wavelets analysis. A method of analyzing nonstationary ultrasonic signals is to consider them as a superposition of a number of localized elementary signals or wavelets; so that wavelet transformation can be used to analyze and identify the various components of an ultrasonic signal. The WT is the correlation between the signal $y(t)$ and a set of basis functions:

$$
T_{m}(a, b)=\int_{y} y(t) \frac{1}{\sqrt{|a|}} \psi \cdot\left(\frac{t-b}{a}\right) d t
$$

where $b$ is the time domain variable and $a$ is the scaling factor which is linked to the frequency domain variable. $\quad \psi(t-b) /(t)$ is the set of daughter wavelets generated from the mother 
wavelet $\psi(t)$ by dilatation or compression operations in time. $\psi(t)$, the mother wavelet, is a function $h(t)$ which satisfies the following admissibility conditions[12]: $\int_{11}^{|H(\omega)|} \frac{\mid H}{\omega} d \omega<+\infty$, where $\omega$ is the angular frequency and $H(w)$ the Fourier transform of $h(t)$. Any continuous function which is band limited and has a zero mean value can thus be used as a mother wavelet. The continuous Wavelet used in this work is the Morlet[13] wavelet that is formed by Gaussian modulation of a complex sinusoid. $h(1)=e^{2} e^{n x}$

The main interest of this analysis is that the time and frequency resolutions are variant : there is for example a very good time resolution at high frequency. Thus, wavelet transform allows details to be exhibited in time and in frequency domains.

\subsection{Wide Band Processing(WBP)}

As defined above, the direct wavelet transform has a high qualitative interest but it needs to be extended to make it robust for accurate time and/or frequency measurements

One of the main limilations of the common velocity estimation methods is that they have good behavior mainly with narrow band signals.

The narrow band assumption [14] for a ultrasound signal in a flowing fluid is expressed as $2 v / c<</ T B$, where $\mathrm{T}$ is the signal dutation and $B$ its bandwidth. This assumption of narrow band processing can be violated either if the velocity of the scatterers is high or if the time-bandwidth product TB is large. In this case, wide band processing must be applied. A wide band signal is also a signal whose fractional bandwidth $\left(B / f_{0}\right)$ is greater than $10 \%$.

In the case of narrow band processing, the reflected ultrasound signal following a transmitted pulse can be approximated by a time delayed and Doppler-shifted replica of the emitted signal: $r(t)=s(t-\tau) e^{i x i}$

In the wide band case, the received signal is approximated by a time-delayed and scaled replica of the source signal [14] :

$r(t)=\frac{1}{\sqrt{|a|}} s\left(\frac{t-\tau}{a}\right) ;$ where $1 /|\sqrt{a}|$ is an energy normalization.

The wide band cross correlation proves to be a useful tool when the wide band hypothesis is taken into account.

Assume $x(t)$ a transmitted pulse and $y(t)$ the received signal, the scaling factor and the time shift between $x$ and $y$ are given by: $\left(s_{m a l}, \tau_{m a x}\right)=\left.\operatorname{Argmax}\left[T_{x y}(s, \tau)\right]\right|_{s . \tau}$ where $T_{x y}$ is the wavelet transform of $y$ with respect to $x$, that is :

$$
T_{n}(x, \tau)=\frac{1}{\sqrt{|s|}} \int x(t) y\left(\frac{t-\tau}{s}\right) d t \text {. For a narrow band signal }
$$

$f_{d}=\left(s_{\text {tudx }}-1\right) f_{\ell}$ where $f_{d}$ is the Doppler frequency linked to the velocity by eq. (I). For a wide band signal $v=c\left(1-s_{\max }\right) /\left(1+s_{\max }\right)$. $\tau_{m a x}$ is related to the time delay between the two signals.

\section{COMPARISON AND DISCUSSIONS}

Two kinds of simulations are performed. In the first one, an analytical Doppler signal is generated with the model[2] described in section 2. Fig.2 shows the wavelet-transform of the Doppler signal. In Fig.3, we show the theoretical velocity and estimated time profile obtained with the previous methods. As it can be seen the higher the velocity, the greater the errors. All the methods presented here track the time profile velocity with good efficiency.

In the second simulation, we just consider the maximum possible velocity of the fluid which is $7.5 \mathrm{~m} / \mathrm{s}$. We assume that a pulse is transmitted toward air point scatter fluid, at $f_{0}=100$ $\mathrm{kHz}$ with a fractional bandwidih of $60 \%$ and backscattered with a delay $\tau=0.14 \mu \mathrm{s}$ with the celerity $c=300 \mathrm{~m} / \mathrm{s}$. IV, WVD and WBP methods are used to estimate the frequency shift and then the velocity. Fig. 4 shows the bias and standard deviation of the frequency estimates on 50 realizations. The shift frequency to be estimated is $5 \mathrm{kHz}$. Note that the classical Doppler technique cannot directly furnish the delay $\tau$.

For the Wide Band method the delay is also obtained. In applications where the ultrasound celerity is unknown, this delay allows to estimate its value. Table I shows the delay and the velocity estimates on 50 realizations, in the case of a high level noise in the measurement system. The bias and the variance are comparable for scale and delay estimates. For low SNR, large errors occur. Correct estimates are obtained around $0 \mathrm{~dB}$. Fig. 4 shows the behavior of the IV, WVD and WBP methods on a larger set of SNR.

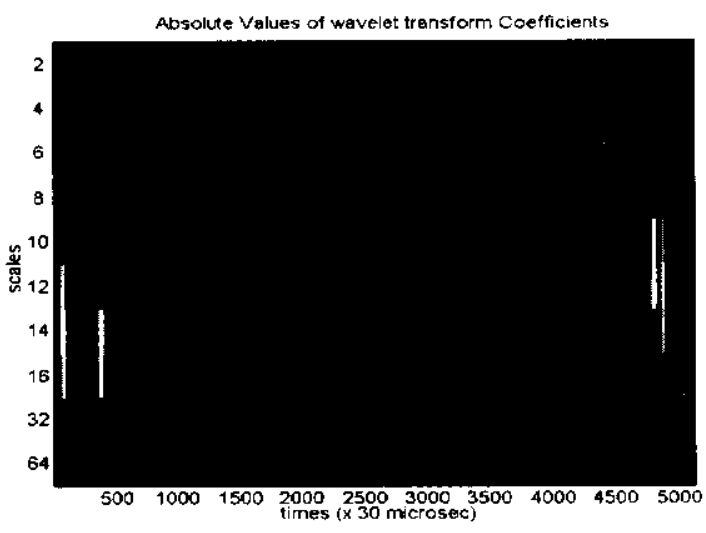

Fig.2. The wavelet transform coefficients of the Doppler signal

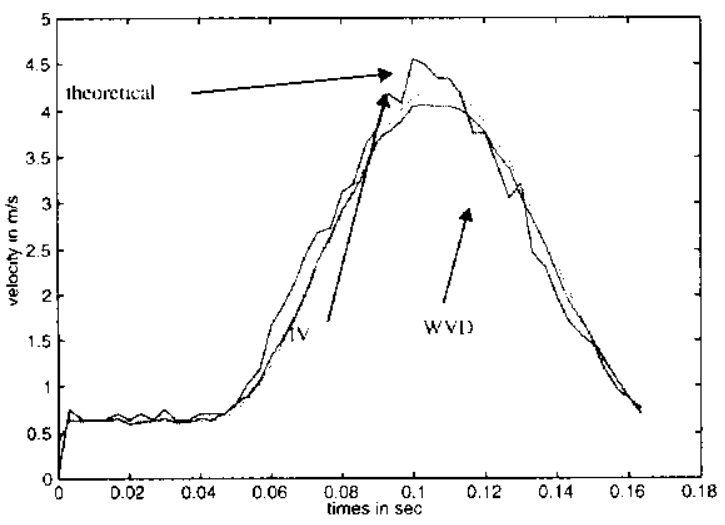

Fig.3. Theoretical and estimated velocity profile. The IV method order is found to be 2 . 


\begin{tabular}{|c|c|c|c|}
\hline $\operatorname{Snr}(\mathrm{dB})$ & -20 & -10 & 0 \\
\hline$\tau(\mu \mathrm{s})$ & 0.03 & 0.04 & 0.11 \\
\hline $\mathrm{v}(\mathrm{m} / \mathrm{s})$ & 75 & 33 & 7.3 \\
\hline
\end{tabular}

Table 1: Time delay and velocity estimates of the second example

As it can be seen, the WBP method is better than the WVD and IV ones in the terms of bias (the variance of IV and WBP methods are similar). However, the WBP method is less suitable for real time estimation. Unlike the WVD, the IV does not need a FFT procedure to be implemented and is thus more accurate when only few data are processed.

\section{CONCLUSION}

Due to the physical parameters of the measurement environment, the ultrasound Doppler signal can be a particularly tricky signal. Three classes of methods are discussed in this paper : a specific IV method which accounts for the possible change of the model order during the estimate process, a Wigner-Ville approach and a wavelet transform. The wide band wavelet method seems to give better qualitative results. Deeper investigation and more exhaustive comparison are now being performed using experimental data.

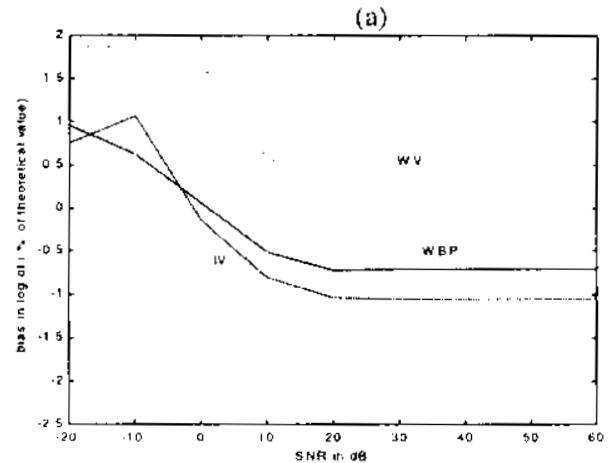

(b)

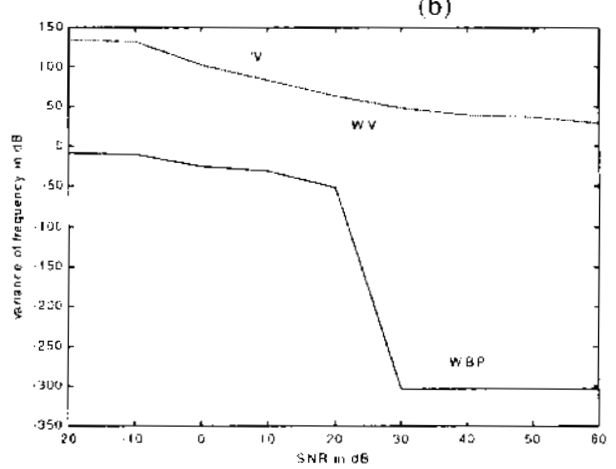

Fig.4. Bias (a) and variance (b) of the frequency estimates with the Wigner Ville distribution (WVD), the Instrumental Variable (IV) and the Wide Band Processing (WBP)

\section{REFERENCES}

[1] J.L.Garbini, F.K.Forster, J.E. Jorgensen: "Measurement of fluid turbulence based on pulsed ultrasound techniques. Part 1\&2. Analysis \& experimental investigation". J.Fluid.Mech., vol. 118 , pp 445-505, 1982.

[2] F. Wendling, S.A. Jones, D.P. Giddens "Simulation of Doppler ultrasound signals, for laminar, pulsatile, non uniform flow ", Ultrasound in Med. \& Biol. vol $18 \mathrm{~N}^{\circ} 2$ pp 179-193, 1992.

[3] W.D.Barber, J.W.Eberhard, S.G.Karr, "A new time domain technique for velocity measurements using Doppler ultrasound", IEEE Trans.Biomed. Eng., vol BME-32, pp $213-$ 229,1985

[4] A. Herment and J.F. Giovannelli, "An adaptive approach to computing the spectrum and mean frequency of Doppler signal", Ultrasonic Imaging, vol 17, N², pp 1-26, 1995.

[5] S.M Kay., S.L.Marple, "Spectrum analysis : a modern perspective", proc. IEEE 69 pp 1380-1419, 1981.

[6] G.J. Birman,"Factorization Methods for Diserete Sequentiel Estimation", New York Academic, 1977

[7] Nui, D. Xiao, D.G. Fisher, "A recursive Algorithme for simultaneous Identification of Model Order and Parameters", IEEE Trans. on Acoust. Sp. and Sig. Proc, vol $38 n^{\circ} 5$ pp 884$886,1990$.

[8] JP. Réménierals, D. Kouame, JM. Gégoire F. Patat. «Multiphase Pipe Flow Velocity measurement in strong colored noisy Doppler ultrasound .» IEEE Ultrasonic Symp. 1996

[9] D. Kouame, J.P. Remenieras, J.F. Roux, A. Ouahabi, M.Lethiecq, "A Modified parametric estimate applied to ultrasound Doppler velocity measurement" Proc. IEEE IMTC, Brussels June 1996

[10] L. Cohen, "Time-Frequency Distributions - A review" Proceeding of the. IEEE vol.77 $n^{\circ} 7,1989$.

[11] M. Basseville et al. "Méthodes temps-fréquence "Traitement du signal supl. vol.9 n¹, 1992

[12] A. Grossmann, R. Kronland-Martinet, "time and scale repretations obtained trough continuous wavelet transforms", Signal Processing $\mathrm{N}^{\circ} 4$, pp. 475-482, 1988.

[13] J. Morlet, G. Arens, I. Fourgeau, D. Giard, «Wave propagation and Sampling theory ", Geophysics, 47, pp 203236,1982 .

[14] Lora C. Weiss, "wavelets and wideband correlation processing", IEEE signal Processing Magazine, pp. 13-32, 1994

[15] O.Rioul. M. Vetterli, "wavelets and signal processing", IEEE Signal Processing Magazine, vol. 8, N²4, pp. 14-38, 199 । 